\title{
Changing Trends in Hyperacute Stroke Treatment and Early Clinical Outcomes Reflecting Recent Improvement in Endovascular Thrombectomy Techniques
}

\author{
Travis William Davidson ${ }^{1}$, Santanu Chakraborty ${ }^{1}$ \\ ${ }^{1}$ University of Ottawa, Faculty of Medicine
}

ABSTRACT

Objective: Ischemic stroke is a major disease causing death and permanent neurological disability with the mainstay treatment being to re-established blood flow as quickly as possible. Recent changes in the efficiency of endovascular devices performing thrombectomies have led to it now being considered the gold standard. Nevertheless, successful endovascular thrombectomy (EVT) requires access to many resources and expertise which may impact its effectiveness. The objective of the current study is to describe the application and utility of EVT in the clinical treatment of ischemic stroke at a single tertiary care hospital.

Methods: This is a descriptive retrospective cohort study from a stroke database. A total of 548 patients diagnosed with an ischemic stroke affecting large cerebral arteries between January 2010 and June 2016 were included. National Institute of Health Stroke Scale (NIHSS) score was used to evaluate stroke severity upon admission. Modified Ranking Scale was used to determine the functional status of patients upon discharge. Successful reperfusion defined as a $\mathrm{TICl}$ score of $2 \mathrm{~B}$ or 3 , age, thrombolytic use and tme-totreatment were all considered for analysis.

Results: Thrombectomies became more frequent $(p<0.001)$ with shorter tme-to-treatment in recent years $(p=0.001)$. A successful thrombectomy has significantly improved functional status at discharge $(p<0.001)$, even when thrombolytics were not given $(p=0.003)$ or when patients were over 80 years old $(p=0.021)$.

Conclusion: The current work provides evidence that this single hospital has increased its frequency of application of EVT for ischemic stroke, leading to a better functional outcome at discharge independent of prior thrombolysis or age.

\section{RÉSUMÉ}

Objectif: L'accident vasculaire cérébral ischémique est une maladie majeure causant la mort et une invalidité neurologique permanente. Le traitement principal consiste à rétablir la circulation sanguine le plus rapidement possible. Des changements récents dans l'efficacité des dispositifs endovasculaires effectuant des thrombectomies ont conduit à ce qu'il soit maintenant considéré comme norme de référence. Néanmoins, le succès de la thrombectomie endovasculaire (TEV) nécessite l'accès à de nombreuses ressources et compétences qui peuvent avoir un impact sur son efficacité. L'objectif de la présente étude est de décrire l'application et l'utilité de la TEV dans le traitement clinique de l'AVC ischémique dans un seul hôpital de soins tertiaires.

Méthodes: Ceci est une étude de cohorte rétrospective descriptive réalisée à partir d'une base de données sur les AVC. Un total de 548 patients ayant reçu un diagnostic d'accident vasculaire cérébral ischémique touchant de grandes artères cérébrales entre janvier 2010 et juin 2016 ont été inclus. Le score du National Institute of Health Stroke Scale (NIHSS) a été utilisé pour évaluer la gravité des AVC à l'admission. Une échelle de classement modifiée a été utilisée pour déterminer l'état fonctionnel des patients à la sortie. Le succès de la reperfusion définie comme un score $\mathrm{TICl}$ de $2 \mathrm{~B}$ ou 3, l'âge, I'utilisation thrombolytique et le délai de traitement ont tous été pris en compte pour l'analyse.

Résultats: Les thrombectomies sont devenues plus fréquentes $(p<0,001)$ avec un délai de traitement plus court ces dernières années $(p=0,001)$. Une thrombectomie réussie a significativement amélioré l'état fonctionnel à la sortie $(p<0,001)$, même lorsque les thrombolytiques n'étaient pas administrés $(p=0,003)$ ou lorsque les patients avaient plus de 80 ans $(p=0,021)$.

Conclusion: Le travail actuel fournit la preuve que cet hôpital seul a augmenté sa fréquence d'application de la TEV pour l'accident vasculaire cérébral ischémique, conduisant à un meilleur résultat fonctionnel à la sortie indépendamment d'une thrombolyse antérieure ou de l'âge.

Keywords: Stroke; Thrombectomy; Ischemic; Hyperacute 
S troke is a major disease with increasing prevalence, currently affecting over an estimated 400000 Canadians, causing death and permanent neurological disability, leading to heavy economic burden from hospitalizations, long-term disability and productivity loss (1).

Blood flow must be re-established as quickly as possible during an ischemic stroke to minimize brain cell death. The use of intravenous clot busting drugs, known as thrombolytics, are approved for the treatment of hyperacute ischemic strokes. The use of thrombolytics alone is traditionally viewed as a risky medical procedure since it may cause or exacerbate an intracerebral hemorrhage or systemic bleeding with potentially deadly side effects; therefore, it is only recommended to offer this treatment if it can be given within 4.5 hour of stroke onset $(2,3)$. These potentially lethal side effects of thrombolytics lead the Canadian Association of Emergency Physicians to adopt a more conservative 3 hour tme limit for thrombolytic use (4).

The use of endovascular devices to remove cerebral blood clots has been a promising method to quickly and effectively restore blood flow following an ischemic stroke with less side effects and more efficiency than thrombolytics. Limitations in advanced neuroimaging, poor recanalization using first generation devices and prolonged tme-to-treatment have led to prior negative randomized controlled studies (5). Recent improvements in the thrombectomy techniques and imaging triage have led to a string of successful clinical trials (6) resulting in endovascular thrombectomies (EVT) being considered the gold standard of care (7) and causing the revision of hyperacute stroke guidelines by the American Stroke Association and Heart \& Stroke Foundation of Canada $(2,8)$. Endovascular thrombectomies have been found to be safe when combined with intravenous thrombolytics and in patients on oral anticoagulants $(9,10)$ and has a larger therapeutic window of 6 hours that could become larger pending more research (2).

Nevertheless, a successful endovascular thrombectomy requires access to many resources which may not be available in all medical centers. These resources include access to advanced neuroimaging, intensive care unit (ICU), angiography suite tme, interventional neuroradiology and preferably a dedicated stroke team. Additionally, the use of endovascular treatment is limited to large-vessel occlusions and is estimated to apply in only $7 \%$ to $13 \%$ of ischemic strokes depending on the permissive criteria (11).
Although there is promising research demonstrating the utility of endovascular thrombectomies, there is a lack of evidence to demonstrate how this has influenced the clinical practice of hyperacute stroke treatment. The objective of the current study is to describe the application and utility of EVT in the clinical treatment of ischemic stroke of large cerebral vessels at a single tertiary care academic hospital.

\section{METHODS}

Study design and participants

We conducted a single center retrospective cohort study from a stroke database consisting of consecutive patients who underwent CT perfusion (CTP) scanning in a 320-multislice Toshiba CT scanner at the Civic Campus of The Ottawa Hospital (TOH) following a stroke code. Our dataset represents a random subset of the stroke patients in the Ottawa Valley region chosen as part of a larger study on CTP in stroke. Suspected acute stroke patients within our catchment area are all redirected to the Civic Campus of $\mathrm{TOH}$ where advanced hyperacute stroke care is provided by a dedicated stroke team. The current study collected clinical data of these stroke patients by performing a chart review from electronic patient record (vOacis) to document their follow-up course and prognosis. Data was collected starting from January 2010 until June 2016.

Patients diagnosed with an ischemic stroke and documented thrombus in the anterior cerebral artery (ACA), middle cerebral artery (MCA), posterior cerebral artery (PCA), vertebral and/or basilar artery were included. There were no exclusion criteria based on age, morbidity, tme-to-treatment or concurrent treatment with intravenous thrombolytics. All data was collected using online, de-identified patient records: the electronic medical record (vOacis) and radiological database (PACS). Approval for this study was obtained from the local REB (OHSN-REB 2009474-01H). Data were collected regarding each patient's presentation, clinical course, diagnostic imaging and thrombectomy results and follow-up.

Institutional acute stroke workflow

For patients presenting with suspected stroke, an Emergency Department physician initiates a stroke code. The dedicated stroke team consists of a neurologists, diagnostic and interventional neuroradiologists, neurological ICU team with support from emergency department physicians, nurses and stroke coordinators. Patients first undergo a neurological exam, NIHSS score calculation, blood glucose levels and an acute stroke 
CT scan protocol. Our acute stroke imaging starts with a noncontrast $\mathrm{CT}$ followed by combined whole brain perfusion and cranial CT angiogram (CTA) acquisition. This is followed by CTA of the neck with a separate bolus of contrast injection. The NIHSS score, the CT scan and the patient health records are then analyzed in order to assess inclusion and exclusion criteria for thrombolytics, with an aim of this assessment occurring within 60 minutes of the stroke code being called, and/or providing a thrombectomy if occlusion is suspected from a CT angiogram (12). Usually the patients will have intravenous thrombolytics as they are being prepared for thrombectomy.

\section{Stroke assessment}

We identified patients with suspected acute stroke less than 6 hours from symptom onset who had CT scans as per our stroke protocol and confirmed thrombus in one of the large intracranial arteries. NIHSS scores of less than 5 were considered mild, 6-13 moderate, and scores over 13 were considered a severe stroke (13). The Modified Ranking Scale (mRS) was used to determine the degree of disability of the stroke patients upon discharge. A score of 0-2 was considered functionally independent while a score of 3-5 was considered functionally dependent, and a score of 6 indicating death (14). Details of treatment received with thrombolytics or endovascular thrombectomies were recorded. A thrombectomy was considered successful if a Thrombolysis In Cerebral Infarction (TICI) score of Grade 2B or 3 was achieved (15). Tme-to-treatment for thrombectomies was considered as the tme interval between tme of stroke onset until groin puncture rounded to the half hour.

\section{Statistical analysis}

Univariate and descriptive statistics were calculated for all study variables. Pearson's correlation was used to determine the relationship between different measures. Differences in continuous measures were assessed using 2-sample t-tests, and categorical measures were assessed using Chi-square test. One-Way ANCOVAs were used to assess differences in continuous variables while controlling for a covariate of stroke severity identified during the correlation analysis. We evaluated the association between thrombectomies and mRS scores using logistic regression analysis while controlling for confounding factors of age, tme-to-treatment, thrombolytic treatment and NIHSS stroke severity. This analysis was compared between 7 different years (2010 - 2016). Post hoc analysis was performed with Bonferroni correction to control for multiple comparisons. All $p$ values were two-tailed, and a value of less than 0.05 was considered statistically significant. All analyses were performed using SPSS software version 17.0 for Windows ${ }^{\circledR}$ (Chicago, IL, USA).

\section{RESULTS}

Year-to-year variability

A total of 1361 patients have undergone the stroke code and CTP scanning at TOH between January 2010 and June 2016. From these patients, 560 were found to have an acute thrombus involving the ACA, MCA, PCA or basilar artery and were included in the analysis. Table 1 summarizes all our results from year-to-year. The number of stroke cases treated in our dataset have increased gradually over the years, except for a spike in strokes in 2011. The average age of all patients was 73 years and $53 \%$ of the participants were females with no significant fluctuations from year-to-year, $\mathrm{p}>0.05$. NIHSS scores were only noted in 525 of our participants, with severe stroke being the most common type of stroke at $37.5 \%$, followed by moderate stroke at $33.7 \%$ and mild strokes at $28.8 \%$. There is some significant year-to-year variability in stroke severity $(F=2.178, p=$ 0.044 ), and post hoc analysis revealed that 2014 had less severe strokes than 2010, 2013, 2015 and 2016, $p<0.05$. There was a total of 29 strokes involving the ACA, 484 involving the MCA, 51 involving the PCA and 31 involving the basilar artery, representing $5.2 \%, 86.4 \%, 9.1 \%$ and $5.5 \%$ of all strokes, respectively. These proportions did not significantly differ between years.

A total of 320 (57.1\%) patients received thrombolytics to treat their strokes. Treating with thrombolytics varied significantly between years $F=2.40, p=0.027$. Post hoc analysis demonstrated that 2014 had significantly less use of thrombolytics compared to 2010, 2015 and 2016, $p<0.05$. Thrombolytics were also more common in 2015 than in 2011. The use of thrombolytics in 2016 was more frequent than 2013 and 2011. The overall $\mathrm{mRS}$, mortality and corresponding functional status of the patients at discharge did not change significantly between years. As shown in Figure 1, thrombectomies became more frequent in recent years; $F=9.295, p<0.001$. Post hoc analysis revealed that both 2013 and 2014 had significantly more thrombectomies than 2010, $p<0.05$, while 2015 and 2016 having significantly more thrombectomies than all other years (2010-2014), $\mathrm{p}<0.05$. Thrombectomy tme-to-treatment has significantly improved over the years, $\mathrm{F}=3.374, \mathrm{p}=0.001$. Tme-to-treatment in 2014 and 2016 were each significantly faster than 2011 and 2012, while 2015 had the fastest tme-to-treatment tmes, being significantly faster than 2011, 2012 and $2013(p<0.05)$. 
Table 1. Summary of demographic and stroke data of all patients.

\begin{tabular}{|c|c|c|c|c|c|c|c|}
\hline & \multicolumn{7}{|c|}{ Year } \\
\hline & 2010 & 2011 & 2012 & 2013 & 2014 & 2015 & 2016 \\
\hline Number & 62 & 106 & 89 & 70 & 78 & 102 & 53 \\
\hline \multirow[t]{2}{*}{ Gender } & o' 32 & $\sigma^{\prime} 48$ & $0^{\prime} 43$ & o' 38 & $0^{2} 38$ & o' 38 & o' 26 \\
\hline & $\$ 30$ & \$ 58 & $\$ 46$ & \$ 32 & \$ 40 & 64 & ๆ 27 \\
\hline \multirow[t]{2}{*}{ Age } & $70.3 \pm$ & $72.8 \pm$ & $72.2 \pm$ & $77.6 \pm$ & $70.4 \pm$ & $74.5 \pm$ & $74.2 \pm$ \\
\hline & 15.6 & 16.5 & 13.3 & 11.8 & 16.5 & 15.4 & 15.5 \\
\hline \multicolumn{8}{|l|}{ NIHSS } \\
\hline Mild & $14(22.5 \%)$ & 31 (29.2\%) & 28 (31.5\%) & $14(20.0 \%)$ & 33 (42.3\%) & 22 (21.6\%) & 9 (17.0\%) \\
\hline Moderate & $18(29.0 \%)$ & 32 (30.2\%) & 24 (27.0\%) & 23 (32.9\%) & 20 (25.6\%) & 36 (35.3\%) & $24(45.3 \%)$ \\
\hline Severe & 22 (35.5\%) & 35 (33.0\%) & 30 (33.7\%) & 31 (44.3\%) & 21 (26.9\%) & 39 (38.2\%) & 19 (35.8\%) \\
\hline Unreported & 8 (12.9\%) & $8(7.6 \%)$ & $7(7.8 \%)$ & $2(2.8 \%)$ & $4(5.1 \%)$ & $5(4.9 \%)$ & $1(1.9 \%)$ \\
\hline \multicolumn{8}{|l|}{$\mathrm{mRS}$} \\
\hline Functional & 24 & 55 & 35 & 30 & 38 & 44 & 26 \\
\hline Dependent & 23 & 36 & 42 & 27 & 28 & 41 & 21 \\
\hline Death & 15 & 15 & 12 & 13 & 12 & 17 & 6 \\
\hline ACA & 5 & 4 & 3 & 2 & 5 & 9 & 1 \\
\hline MCA & 50 & 95 & 70 & 63 & 68 & 90 & 48 \\
\hline PCA & 8 & 9 & 12 & 5 & 9 & 5 & 3 \\
\hline Basilar & 5 & 6 & 9 & 2 & 1 & 6 & 2 \\
\hline tPA & 40 & 53 & 49 & 37 & 37 & 66 & 38 \\
\hline Thrombectomy & 2 & 9 & 8 & 11 & 14 & 32 & 21 \\
\hline Unsuccessful & 0 & 2 & 0 & 1 & 1 & 5 & 2 \\
\hline Time-to-treat & $3.0 \pm 2.8$ & $5.8 \pm 6.5$ & $5.4 \pm 2.6$ & $4.1 \pm 1.2$ & $3.6 \pm 2.3$ & $2.6 \pm 0.9$ & $3.7 \pm 2.4$ \\
\hline
\end{tabular}

Continuous data were reported with the mean and standard deviation (SD). Categorical data were summarised as frequency $(n)$ and percentage (\%).

Predictors of post-stroke functional outcome

Pearson's correlation found that better functional status is related to lower age $r=0.300, p<0.001$, milder stroke severity $r=0.409, p<0.001$, thrombectomies $r=-0.11, p=0.009$, successful thrombectomies $r=-0.165, p<0.001$ and reduced tme-totreatment $r=0.207, p=0.042$. Functional status was not related to the use of thrombolytics ( $p>0.05$ ) and was not used in the following linear regression analysis. A linear regression was calculated to predict $\mathrm{mRS}$ scores based on age, stroke severity and thrombectomy. A significant regression equation was found; $\mathrm{F}=81.105, \mathrm{p}<0.001$, with an $\mathrm{R} 2$ of 0.318 . It was found that age $(\beta=0.245, p<0.001)$, thrombectomy $(\beta=-0.210, p<0.001)$ and stroke severity $(\beta=0.475, p<0.001)$ were each significant predictors of $\mathrm{mRS}$ scores.
When including only recent patients after 2015 and only successful thrombectomies, the significant regression; $F=39.871$, $\mathrm{p}<0.001$; had an improved $\mathrm{R} 2$ of 0.452 . With the stroke severity being the strongest significant predictor of $m R S$ scores $(\beta=0.53$, $p<0.001)$, followed by successful thrombectomy $(\beta=-0.424$, $p<0.001)$ then the age of the patient $(\beta=0.21, p=0.002)$.

Efficacy of thrombectomies on functional status

A successful thrombectomy has significantly improved functional status at discharge $\mathrm{t}=3.94, \mathrm{p}<0.001$, as shown in Figure 2. Specifically, thrombectomies were found to be a successful treatment for eligible patients with MCA strokes $t=4.079, p$ $<0.001$, but the 4 patients with basilar strokes who received a thrombectomy were not shown to have an improved func- 


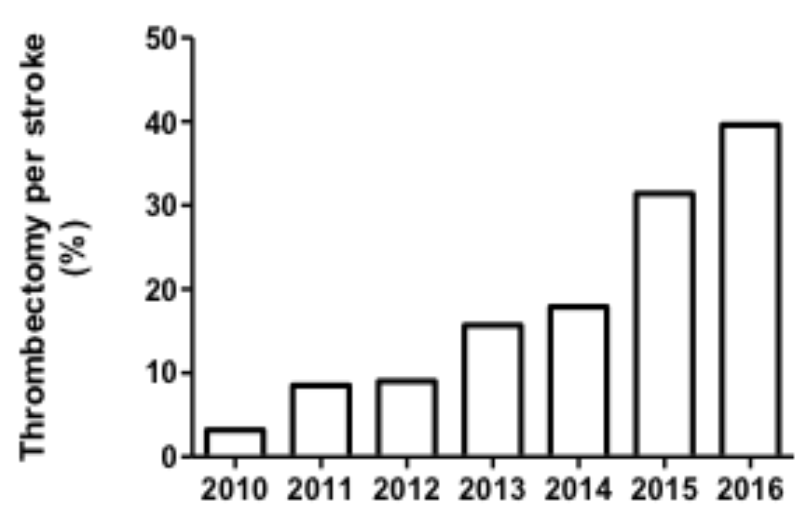

Figure 1. The percentage of patients with ischemic strokes who received thrombectomies according to the year the patient was admitted.

tional status compared to the other basilar strokes patients; $\mathrm{t}=0.167, \mathrm{p}=0.869$.

The 11 cases of thrombectomies with a tme-to-treatment of 6 hours and over were found to not significantly affect functional status, $t=0.922, p=0.357$, but it almost reached significance when controlling for stroke severity, $F=3.827, p=0.051$.

When controlling for stroke severity, thrombectomies were shown to be an effective treatment for ischemic strokes in a subset of patients who did not receive thrombolytics; $F=9.181$, $\mathrm{p}=0.003$.

\section{Efficacy of thrombolytics on functional status}

The application of thrombolytics was not found to significantly improve functional status at discharge; $t=-1.243, p=0.214$, even when controlling for stroke severity; $F=3.581, p=0.059$. However, thrombolytics were found to significantly improve functional status when excluding mild strokes; $t=2.348, p=0.019$.

Efficacy of hyperacute ischemic stroke treatment in older patients

In addition to be related to functional outcome, age was found to be correlated to stroke severity $r=0.124, p=0.004$. In patients over 80 years of age, thrombectomies were only found to significantly improve functional outcome when controlling for the covariate of stroke severity; $F=5.393, p=0.021$. The application of thrombolytics in this age group was found to be associated with significantly worse functional outcomes; $t=-2.639$,

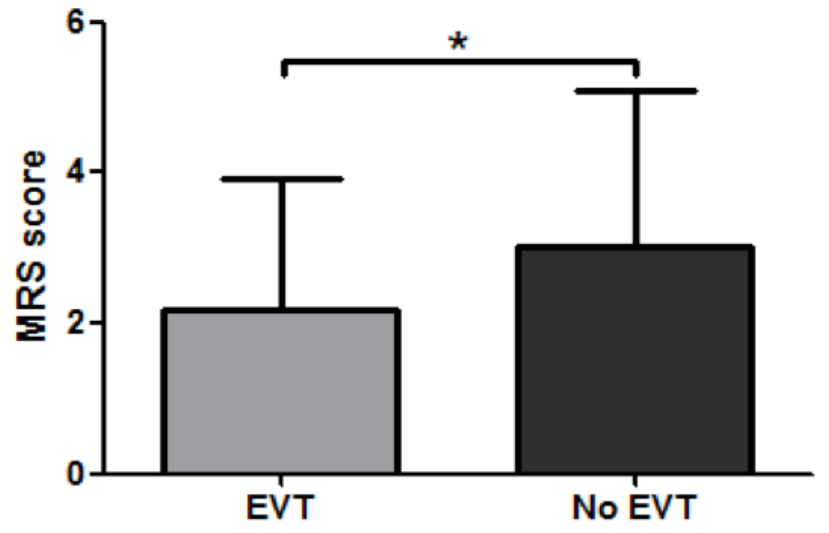

Figure 2. mRS score depending on a successful endovascular thrombectomy (EVT) and without the treatment (No EVT). " represents $p<0.01$.

$\mathrm{p}=0.009$; however, this propensity disappeared when controlling stroke severity; $F=1.127, p=0.290$.

\section{DISCUSSION}

We have provided preliminary evidence that the recent advancements in the use of thrombectomy as a treatment of hyperacute ischemic stroke has significantly influenced clinical practice in our center. Our main finding of the current descriptive study is that EVTs at the Civic Campus of TOH have greatly increased in use in recent years, and its application is associated with a better clinical outcome score at discharge.

\section{Year-to-year variability}

Age, mortality and cerebral artery involved did not change with year. The descriptive nature of the current study has led to a lack of uniformity in our patients between years, such as the increase in participants in 2011, a reduction in stroke severity and the concomitant thrombolytic use in 2014. These changes could be due to random fluctuation in the patients treated at $\mathrm{TOH}$. Our prevalence of thrombolytics are similar to other reports, where there can be a large proportion of patients who do not receive thrombolytics for various inclusion or exclusion criteria (16). The increase use of thrombolytics in 2015 and 2016 could partially be caused by the increased consultations to $\mathrm{TOH}$ for thrombectomies in eligible participants around the Ottawa Valley and better organized acute stroke care in the catchment area. 
Prevalence of thrombectomies

There has been a dramatic increase in the percentage of hyperacute stroke patients treated with thrombectomies in recent years, from 3.2\% of strokes in 2010 to $39.6 \%$ of cases in 2016 as depicted in Figure 1. The percentage of treated cases is far above the predicted $13 \%$ of all ischemic strokes predicted (11). Despite this high prevalence, there is no change in proportion of cerebral artery involved or chances of performing a successful thrombectomy. This could be partially explained by the fact that thrombectomies can only be performed in large cerebral arteries and we only included patients with ischemic strokes where large cerebral arteries were involved. Additionally, there is evidence for increased use and reduced tme-to-treatment of thrombectomies in 2014; however, this becomes much more apparent in 2015 coinciding with the successful clinical trials and new strokes treatment guidelines $(6,8)$.

\section{Predictor of functional outcome}

Consistent with previous reports, we have found that the strongest predictor of functional outcomes following stroke is the age of the patient and stroke severity (17). We also found that thrombectomies and tme-to-treatment of the thrombectomy were significantly related to functional outcome; however, the application of thrombolytic's was not related to functional outcome. A linear regression demonstrated that around $32 \%$ of all variability in functional outcome following ischemic stroke can be predicted using stroke severity, age and the utilisation of a thrombectomy. When we repeated this linear regression for ischemic strokes in 2015 and 2016, its predictive power increased to explain 45.2\% of all variability, and the utilisation of a successful thrombectomy was a better predictor than age.

Functional outcomes following a thrombectomy

A successful thrombectomy was found to be an effective treatment to improve functional outcome following an ischemic stroke even in the absence of thrombolytics and tended towards being efficient even after the suggested 6-hour tme limit (2). This is in accordance with the recent meta-analysis concluding that EVT will benefit most patients with an eligible acute ischemic stroke even if they are over the age of 80 years of age or not eligible for thrombolytics (18). Specifically, this meta-analysis found that the number needed to treat with EVT was 2.6 to reduce the mRS score by at least 1 (18). Despite this recent overwhelming evidence favoring EVT, these findings have not been noted in studies prior to $2015(19,20)$.

Specifically, we found an overall effect that thrombectomy led to a lower mRS score at discharge. Although thrombectomies were found to be therapeutically effective in 2012 and 2014, it became much more apparent in 2015 and 2016 as seen in

Table 2. This is likely due to the increased efficiency in providing thrombectomies to ischemic stroke patients as shown by a reduce tme-to-treatment, which has important implications on functional outcomes (21).

Although the thrombectomy was found to be an effective treatment for select patients with ischemic strokes involving the MCA, it was not found to be effective in improving the functional outcome with basilar artery occlusion. The limited number of cases involving the basilar artery thrombus may not be representative sample for this population since a recent meta-analysis has found that endovascular reperfusion techniques improve functional outcome after basilar artery occlusion (22).

The subset of adults over 80 years of age have also been found to benefit from mechanical thrombectomy as a treatment for ischemic stroke, suggesting that there might be no upper age limit for the utility of thrombectomies. This is in accordance with recent research suggesting that patients older than 70 years of age benefit the most from successful reperfusion following mechanical thrombectomy (23).

Limitations

There are clear limitations to the current study such as the descriptive nature of this study, which prevents any conclusive interpretations of the true efficacies of the observed treatment. There is no short- or long-term follow-up of our stroke patients. We only used a subset of patients who had advanced stroke CT scanning, possibly creating the potential for selection bias. Our stroke database possesses limited health information that could influence stroke treatment or functional outcome such as atrial fibrillation, diabetes, anticoagulant treatment or history of previous strokes (24). We also did not include any sub-analysis of the different endovascular devices used for the thrombectomy procedures where the newer devices have been found to have improved rapidity and degree of revascularization (25).

\section{CONCLUSION}

The current descriptive paper provides evidence that the Civic Campus of $\mathrm{TOH}$ has increased its frequency of application of EVT for the treatment of new hyperacute stroke. These 
Table 2. Functional status evaluated using $\mathrm{mRS}$ scores at discharge in patients who received different ischemic stroke treatments.

\begin{tabular}{|c|c|c|c|c|c|c|c|}
\hline \multirow{2}{*}{$\begin{array}{l}\text { Treatment } \\
\text { group }\end{array}$} & \multicolumn{6}{|c|}{ Year } & \multirow[b]{2}{*}{2016} \\
\hline & 2010 & 2011 & 2012 & 2013 & 2014 & 2015 & \\
\hline No treatments & $\begin{array}{c}2.2 \pm 2.4 \\
(22)\end{array}$ & $\begin{array}{c}2.5 \pm 1.6 \\
(52)\end{array}$ & $\begin{array}{c}2.7 \pm 2.1 \\
(38)\end{array}$ & $\begin{array}{c}2.9 \pm 2.2 \\
(33)\end{array}$ & $\begin{array}{c}2.8 \pm 2.1 \\
(37)\end{array}$ & $\begin{array}{c}3.5 \pm 2.2 \\
(32)\end{array}$ & $\begin{array}{c}3.0 \pm 1.8 \\
\text { (11) }\end{array}$ \\
\hline Thrombolytics & $\begin{array}{c}3.9 \pm 2.0 \\
(40)\end{array}$ & $\begin{array}{c}3.2 \pm 2.0 \\
(53)\end{array}$ & $\begin{array}{c}3.1 \pm 2.0 \\
(49)\end{array}$ & $\begin{array}{c}2.8 \pm 2.3 \\
(37)\end{array}$ & $\begin{array}{c}2.6 \pm 2.3 \\
(37)\end{array}$ & $\begin{array}{c}2.8 \pm 1.9^{*} \\
(66)\end{array}$ & $\begin{array}{c}2.5 \pm 2.0 \\
(21)\end{array}$ \\
\hline Thrombectomy & $\begin{array}{c}6.0 \pm 0.0 \\
\text { (2) }\end{array}$ & $\begin{array}{c}3.3 \pm 1.8 \\
(9)\end{array}$ & $\begin{array}{c}2.6 \pm 2.1 \\
(8)\end{array}$ & $\begin{array}{c}2.8 \pm 2.0 \\
(11)\end{array}$ & $\begin{array}{c}2.0 \pm 1.8 \\
(14)\end{array}$ & $\begin{array}{c}2.5 \pm 1.9^{* *} \\
(32)\end{array}$ & $\begin{array}{c}1.8 \pm 1.5^{* *} \\
(38)\end{array}$ \\
\hline All patients & $\begin{array}{c}3.3 \pm 2.3 \\
(62)\end{array}$ & $\begin{array}{c}2.9 \pm 1.8 \\
(106)\end{array}$ & $\begin{array}{c}3.0 \pm 2.0 \\
(89)\end{array}$ & $\begin{array}{c}2.9 \pm 2.2 \\
(70)\end{array}$ & $\begin{array}{c}2.7 \pm 2.2 \\
(78)\end{array}$ & $\begin{array}{c}3.0 \pm 2.0 \\
(102)\end{array}$ & $\begin{array}{c}2.6 \pm 1.9 \\
(53)\end{array}$ \\
\hline
\end{tabular}

Data was reported as mean \pm standard deviation (SD) with frequency reported underneath (n). ANCOVAs were conducted controlling for stroke severity to evaluate the effect of specified treatment on mRS scores. ${ }^{*}$ indicates $p<0.05 ;{ }^{* *}$ indicates $\mathrm{p}<0.001$.

thrombectomies have led to a better functional outcome at discharge for those who were eligible for this treatment despite the absence of prior thrombolysis or advanced age.

\section{REFERENCES}

1. Krueger H, Koot J, Hall RE, O'Callaghan C, Bayley M, Corbett D. Prevalence of individuals experiencing the effects of stroke in Canada. Stroke. 2015; 46(8): 2226-31.

2. Powers WJ, Derdeyn CP, Biller J, et al. 2015 american heart association/american stroke association focused update of the 2013 guidelines for the early management of patients with acute ischemic stroke regarding endovascular treatment: A guideline for healthcare professionals from the american heart association/american stroke association. Stroke. 2015;46(10):302035.

3. Harris DR, Lang ES, Perry JJ, Morrison LJ. Treatment of stroke in canadian emergency departments: Tme to be leaders. CJEM. 2017;19(1)47-9.

4. Harris D, Hall C, Lobay K, et al. Canadian association of emergency physicians position statement on acute ischemic stroke. CJEM. 2015;17(2):21726.

5. Maingard J, Yan B. Future directions for intra-arterial therapy for acute ischaemic stroke: Is there life after three negative randomized controlled studies? Interv Neurol. 2014;2(3):97-104.

6. Beadell NC, Bazan T, Lutsep H. The year embolectomy won: A review of five trials assessing the efficacy of mechanical intervention in acute stroke. Curr Cardiol Rep. 2015;17(11):102

7. Palaniswami M, Yan B. Mechanical thrombectomy is now the gold standard for acute ischemic stroke: Implications for routine clinical practice. Interv Neurol. 2015;4(1-2):18-29.

8. Casaubon LK, Boulanger JM, Blacquiere D, et al. Canadian stroke best practice recommendations: Hyperacute stroke care guidelines, update 2015. Int J Stroke. 2015;10(6):924-40.

9. Tsivgoulis G, Katsanos AH, Mavridis D, Magoufis G, Arthur A, Alexandrov AV. Mechanical thrombectomy improves functional outcomes independent of pretreatment with intravenous thrombolysis. Stroke. 2016;47(6):1661-4.

10. Purrucker JC, Wolf $M$, Haas K, et al. Safety of endovascular thrombectomy in patients receiving non-vitamin $\mathrm{k}$ antagonist oral anticoagulants. Stroke. 2016:47(4):1127-30.

11. Chia NH, Leyden JM, Newbury J, Jannes J, Kleinig TJ. Determining the number of ischemic strokes potentially eligible for endovascular thrombectomy: A population-based study. Stroke. 2016;47(5):1377-80.

12. Chakraborty S, Ross J, Hogan MJ, Dowlatshahi D, Stotts G. Beating the clock: Tme delays to thrombolytic therapy with advanced imaging and impact of optimized workflow. J Stroke Cerebrovasc Dis. 2015;24(6):1270-5.

13. Schlegel D, Kolb SJ, Luciano JM, et al. Utility of the nih stroke scale as a predictor of hospital disposition. Stroke. 2003;34(1):134-7.

14. van Swieten JC, Koudstaal PJ, Visser MC, Schouten HJ, van Gijn J. Interobserver agreement for the assessment of handicap in stroke patients. Stroke. 1988;19(5):604-7.

15. Fugate JE, Klunder AM, Kallmes DF. What is meant by "tici"? AJNR Am J Neuroradiol. 2013;34(9):1792-7.

16. Hills NK, Johnston SC. Why are eligible thrombolysis candidates left untreated? Am J Prev Med . 2006;31 (6 Suppl 2):S210-6.

17. Harvey RL. Predictors of functional outcome following stroke. Phys Med Rehabil Clin N Am 2015;26(4):583-98.

18. Goyal M, Menon BK, van Zwam WH, et al. Endovascular thrombectomy after large-vessel ischaemic stroke: A meta-analysis of individual patient data from five randomised trials. Lancet. 2016;387(10029):1723-31.

19. Ciccone A, Valvassori L, Nichelatti M, et al. Endovascular treatment for acute ischemic stroke. N Engl J Med. 2013;368(5):904-13.

20. Kidwell CS, Jahan R, Gornbein J, et al. A trial of imaging selection and endovascular treatment for ischemic stroke. N Engl J Med. 2013;368(10):914-23.

21. Gerber J, Puetz V, Bodechtel U, Kitzler H, von Kummer R, Linn J. Endovascular treatment of ischaemic stroke patients - new evidence and old challenges. Vasa. 2016;45(4):267-74.

22. Kumar G, Shahripour RB, Alexandrov AV. Recanalization of acute basilar 
artery occlusion improves outcomes: A meta-analysis. J Neurointerv Surg 2015;7(12):868-74.

23. Slater LA, Coutinho JM, Gralla J, et al. Tici and age: What's the score? AJNR Am J Neuroradiol. 2016;37(5):838-43.

24. Esenwa C, Gutierrez J. Secondary stroke prevention: Challenges and solutions. Vasc Health Risk Manag. 2015;11:437-50.

25. Przybylowski CJ, Ding D, Starke RM, Durst CR, Crowley RW, Liu KC. Evolution of endovascular mechanical thrombectomy for acute ischemic stroke. World J Clin Cases. 2014;2(11):614-22. 